\title{
REM Sleep Behavior Disorder Accompanying Arachnoid Cyst: A Case Report
}

\author{
Erhan $\mathrm{AkıncI}^{1^{*}}$, Ali Samancıoğlu² \\ ${ }^{1}$ Buca State Hospital, Psychiatry Clinic, Izmir, Turkey \\ ${ }^{2}$ Buca State Hospital, Neurosurgery Clinic, Izmir, Turkey
}

\begin{abstract}
REM sleep behavior disorder (RBD) is a parasomnia which is characterized by a loss of the normal skeletal muscle atonia associated with REM sleep with dream enactment behavior that is generally violent in nature. Serious injury to the patient or the bed partner can result from RBD episodes. Neuroanatomical and physiological integrity of structures in the brainstem is important for the continuity of REM atonia. Brain anomalies and lesions that affect the brainstem may play a causal role in the pathophysiology of RBD. In this case, the presentation of a 73-year-old woman who is diagnosed with RBD accompanying arachnoid cyst in posterior fossa was made.
\end{abstract}

Keywords: parasomnia, REM sleep, arachnoid cyst

\section{INTRODUCTION}

According to the International Classification of Sleep Disorders (ICSD), REM (rapid eye movement) sleep behaviour disorder is classified under the title of parasomnias. RBD is characterized by abnormal behaviours appearing during REM sleep that may cause injury to the patient or the bed partner. RBD occurs when there is loss of skeletal muscle atonia during REM sleep resulting in dream content related vocalization and/or complex motor activities. These episodes may occur rarely or up to several times nightly. Patients have the risk of sleep related injury to self or bed partner due to

*Correspondence: drerhanakinciayahoo.com

Buca Seyfi Demirsoy Devlet Hastanesi, Kozağaç Mah. Özmen Cad. No: 147 Buca-Izmir, Turkey

Tel: +90-0232-4443508

\section{Sleep and Hypnosis \\ Submit your manuscript at www.sleepandhypnosis.org}

abnormal or disruptive behaviors such as punching, grabbing, biting or kicking. These behaviors can result in serious and sometimes life-threatening injuries (Aurora et al, 2010; AASM, 2014).

It is a male predominant disorder that usually appears after age 50 years. It has been reported that $87.5 \%$ of this case series consist of elderly men (Schenk et al, 1993). Although the frequency of the disease is not known exactly, prevalence estimates are $0.38-0.5 \%$ in the general population (Aurora et al, 2010). Pathophysiology of RDB has not completely been understood. The pathophysiology of RBD is assumed to correspond to the findings from an animal model of RBD. Damage to the atonia areas in the pons can result in REM sleep without atonia. In animal models of RBD, experimentally induced pontine tegmental lesions in cats can result in loss of REM atonia and body movements during REM sleep (Sakai et al., 1979; Schenck and Mahowald, 2002). RBD may be idiopathic or secondary. Secondary RBD can be related to neurodegenerative disorders such as synucleinopathies, other neurological disorders or medications (AASM, 2014). 
According to International Classification of Sleep Disorders (ICSD-3) RBD diagnostic criteria is determined as follows:

A. Repeated episodes of sleep related vocalization and/or complex motor behaviors.

B. These behaviors are documented by polysomnography to occur during REM sleep or, based on clinical history of dream enactment, are presumed to occur during REM sleep.

C. Polysomnographic recording demonstrates REM sleep without atonia.

D. The disturbance is not better explained by another sleep disorder, mental disorder, medication or substance use.

Criteria A - D must be met for the diagnosis of RBD (AASM, 2014).

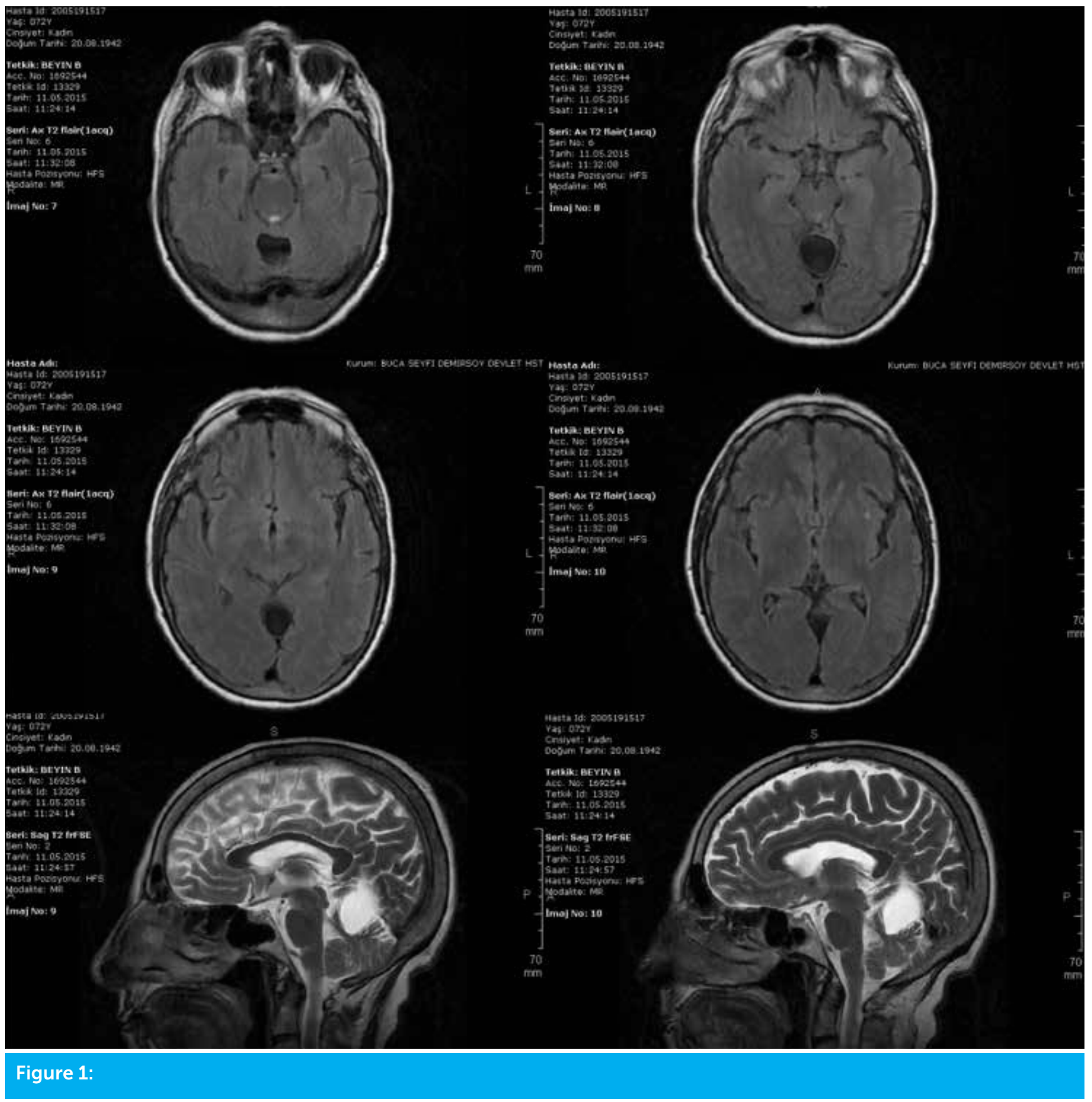




\section{CASE REPORT}

73 year-old married woman patient lives with her family. She was taken to the polyclinic by her daughter with the complaints of screaming, punching, kicking and getting out of the bed during sleep. The patient is reported to have symptoms like punching the wall, jumping out of bed and hitting herself. Complaints have been continuing for around one and a half year and approximately once or twice a week. Complaints usually occur towards the morning. The patient is reported to remember her dreams and actions completely and share her experience with her relatives. There were not any known features in her medical history. The patient did not describe any psychiatric condition except the dreams and actions she had at night. There was no history of alcohol use. She did not use any drugs or active drugs. No severe psychiatric or neurological disease was described in her family.

Her physical and neurological examinations were normal. No significant psychopathology was observed except the mild depressive complaints in a period of around two months in her psychiatric examination. Her routine electroencephalography was normal. No findings were determined in her polysomnography except for the phasic activity increase in the chin electromyography during REM sleep. Cranial magnetic resonance imaging (MRI) revealed the presence of an arachnoid cyst in the middle line with the sizes of $25 \times 21 \times 26 \mathrm{~mm}$ at the level of tentorium (Fig. 1).

Night clonazepam $0.25 \mathrm{mg} /$ day treatment was initiated considering RBD in the patient. It was observed in the follow-ups that she benefitted from the treatment considerably and complaints were completely removed.

\section{DISCUSSION}

The active paralysis is observed in all of the voluntary muscles except for the respiratory and

\section{References}

American Academy of Sleep Medicine (2014) International classification of sleep disorders, 3rd ed. Darien, IL: American Academy of Sleep Medicine. extraocular muscles during the normal REM sleep. In $\mathrm{RBD}$, polysomnography demonstrates intermittent loss of REM atonia during REM sleep. The EEG of REM sleep is similar to the EEG of active wakefulness and there is generalized atonia of voluntary muscles during REM sleep (Yılmaz, 2011). In this way, the person is prevented from dream enactment behaviour. Neuroanatomical and physiological integrity of structures in the brainstem is important for the continuity of REM atonia. Brain anomalies and lesions including subarachnoid hemorrhage, ischemic cerebrovascular disease, and brainstem neoplasms that affect the brainstem may play a causal role in the pathophysiology of RBD (Boeve et al., 2007; Aurora et al., 2010). Arachnoid cysts can appear at any location in the cranium. Arachnoid cysts can cause many different neuropsychiatric symptoms depending on their location. All of these effects and symptoms are caused either by compression of the cyst on the surrounding brain tissue or interference with the functions of the surrounding neurologic structures (da Silva et al. 2007; Wester, 2008).

The RBD diagnosis is associated with the arachnoid cyst since the case is female and the patient does not possess any other sleep disorders or psychological disorders and it cannot be explained by any drug or other substance use and posterior fossa arachnoid cyst with atypical location which has the risk of effecting the structures in the brain stem is available. The patient fully benefitted from the low dose of clonazepam treatment.

$\mathrm{RBD}$ is not a simple parasomnia and may be associated with many organic statuses. Diagnostic approaches for RBD therefore must be multifaceted. In line with the opinion of importance of the organic cases in RBD, the report was presented for the purpose of providing data for the researchers of this case.

Aurora, N. R., Zak, R. S., Maganti, R. K. et al (2010) Best Practice Guide for the Treatment of REM Sleep Behavior Disorder (RBD). Journal of Clinical Sleep Medicine, 6, 85-95. 
Boeve, B. F., Silber, M. H., Saper, C. B. et al (2007) Pathophysiology of REM sleep behaviour disorder and relevance to neurodegenerative disease. Brain, 130, 2770-2788.

da Silva, J. A., Alves, A., Talina, M., Carreiro, S., Guimarães, J., Xavier, M. (2007) Arachnoid cyst in a patient with psychosis: Case report. Annals of General Psychiatry, 28;6:16.

Sakai, K., Sastre, J. P., Salvert, D. et al (1979) Tegmentoreticular projections with special reference to the muscular atonia during paradoxical sleep in the cat: an HRP study. Brain Research, 176, 233-254.
Schenk, C. H., Hurwitz, T. D., Mahowald, M. W. (1993) REM sleep behaviour disorder: an update on a series of 96 patients and a review of the world literature. Journal of Sleep Research, 2, 224231.

Schenck, C. H., Mahowald, M. W. (2002) REM Sleep Behavior Disorder: Clinical, Developmental, and Neuroscience Perspectives 16 Years After its Formal Identification in SLEEP. Sleep, 25, 120-138. Wester, K. (2008) Intracranial arachnoid cysts - do they impair mental functions? Journal of Neurol, 255,1113-1120.

Yılmaz, H. (2011) Parasomniler. In H Kaynak, S Ardıç (Ed.), Uyku Fizyolojisi ve Hastalıkları (pp. 333-348). İstanbul, Nobel Matbaacılık. 